\title{
Electron Crystallography in the Age of Pixelated Detectors: From Crystalline /Amorphous Organic Structural Models and Phase Mapping of Beam Sensitive Materials
}

\author{
S Nicolopoulos ${ }^{1 *}$, A Gomez $^{1}$ and PP Das ${ }^{1}$ \\ 1. NanoMEGAS SPRL, Brussels, Belgium. \\ * Corresponding author: info@nanomegas.com
}

New photon counting pixelated detectors may record ED patterns as a two-dimensional (2D) image with a high-speed frame rate ( $>1000 \mathrm{fps}$ ). The use of pixelated detectors has significant advantages over conventional CCD detectors, due to the absence of electronic detection noise, meaning that the statistics are much cleaner and more interpretable at lower beam doses. Applications of such detectors include high resolution image reconstruction of a specimen from ED pattern by using ptychography application $[1]$.

We

have used such pixelated detector (Timepix) [2] to work under extremely low doses (reduced up to $0.001 \mathrm{e} / \AA 2 / \mathrm{sec}$ ) and record useful PED (precession electron diffraction) [3] patterns for electron diffraction tomography work on beam sensitive pharmaceutical materials, even without use of cooling techniques. Electron diffraction data were obtained from 50-150 nm size crystals working in STEM mode to avoid beam damage. This way unit cell \& crystal structure has been determined from various organic structures (Carbamazepine, Nicotinic acid and more recently active pharmaceutical ingredients Ramelteon and Tolvaptan) where atomic positions could be located with picometer level precision [4]. In all cases structure models from electron crystallography match well with crystal structures derived from single crystal X-Ray data (Figure 1).

Using same type of pixelated detector, we have also analysed beam sensitive amorphous compounds (organic pharmaceuticals and its excipients) at RT by collecting ED patterns and generating electron Pair Distribution Function (e-PDF) (with $Q$ values up to $12-15 \AA^{-1}$ ) for amorphous structure identification/fingerprinting; as result of our work, the obtained e-PDF data from a number of reference amorphous organic pharmaceuticals match well with Synchrotron X-Ray PDF obtained results on the same compounds (Figure 2).

On the other hand, TEM based automated 4D STEM scanning orientation/phase map technique (ASTAR) [5] has been applied in combination with pixelated detectors (Medipix) to reveal accurate phase mapping in various mixtures of amorphous /crystalline organic materials, to monitor accurately recrystallization effects at nanoscale. We have been able to obtain good quality ED patterns from crystalline areas (Figure 3) from reference ASD (amorphous solid dispersion mixtures) where we could map in detail amorphous \& crystalline areas with $10 \mathrm{~nm}$ resolution.

\section{References:}

[1] Y Jiang et al., Nature 559 (2018), p. 343.

[2] E van Genderen et al., Acta Crystallogr. A 72 (2016), p. 236.

[3] R Vincent and PA Midgley, Ultramicroscopy 53 (1994), p. 271.

[4] PP Das et al., Org. Process Res. Dev. 22 (2018), p. 1365. 
[5] EF Rauch et al., Zeit fur Krist, 225 (2010), p. 103.
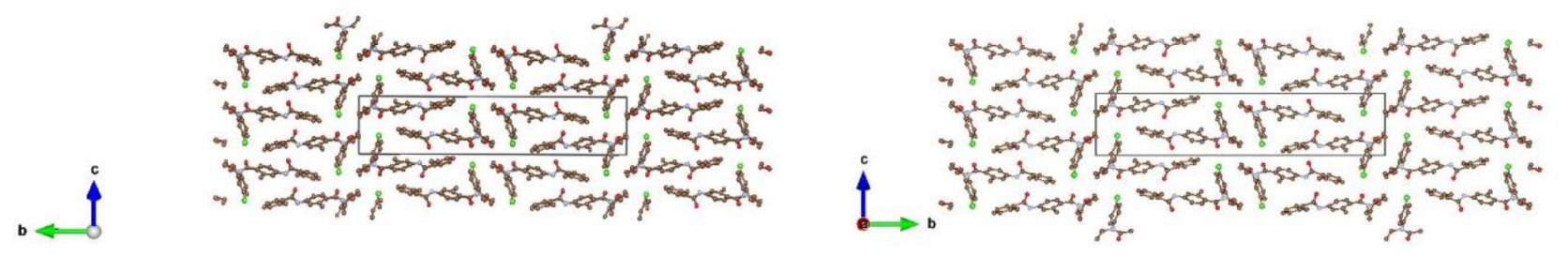

Figure 1. (Left) Tolvaptan API structure as revealed from electron diffraction tomography, (Right) same structure solved by X-Ray diffraction; both models match well.
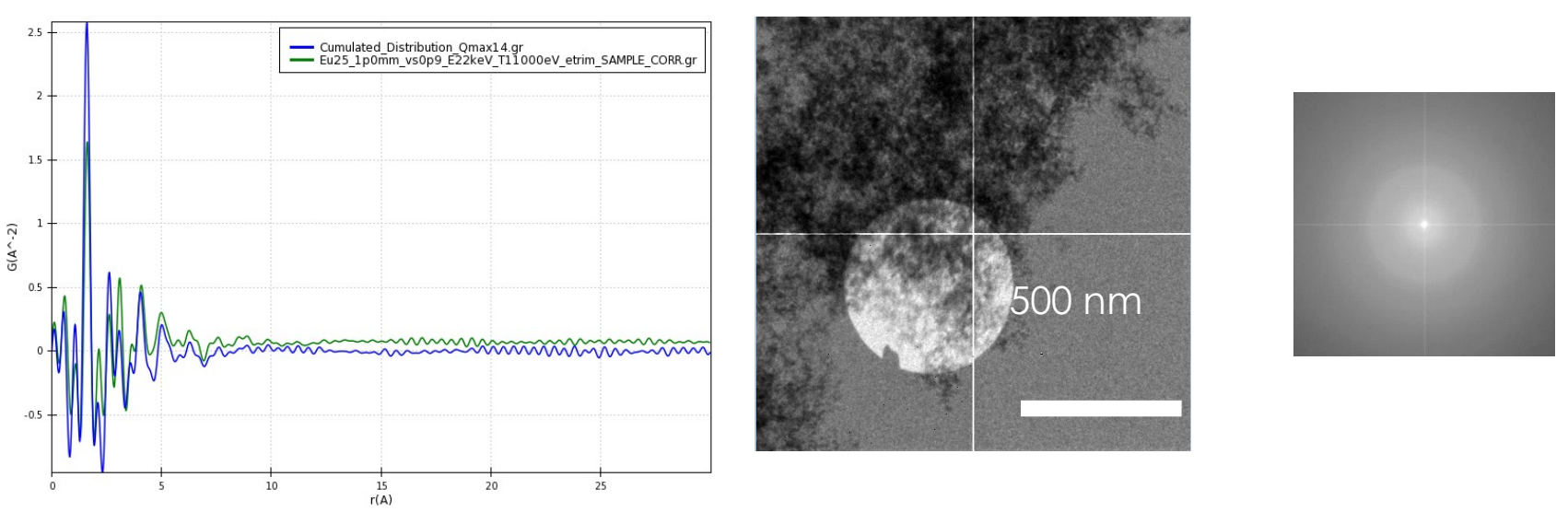

Figure 2. (Left) Comparison of e-PDF (blue colour) and XRD-PDF (Synchrotron, green colour) for Aerosil excipient $\left(Q_{\text {MAX }}=14.0 \AA^{-1}\right.$, e Dose cumulated $\left.=1.08 \mathrm{e}^{-} / \AA^{2}\right)$, (Middle) TEM image of aerosil crystals, (Right) amorphous ED pattern.
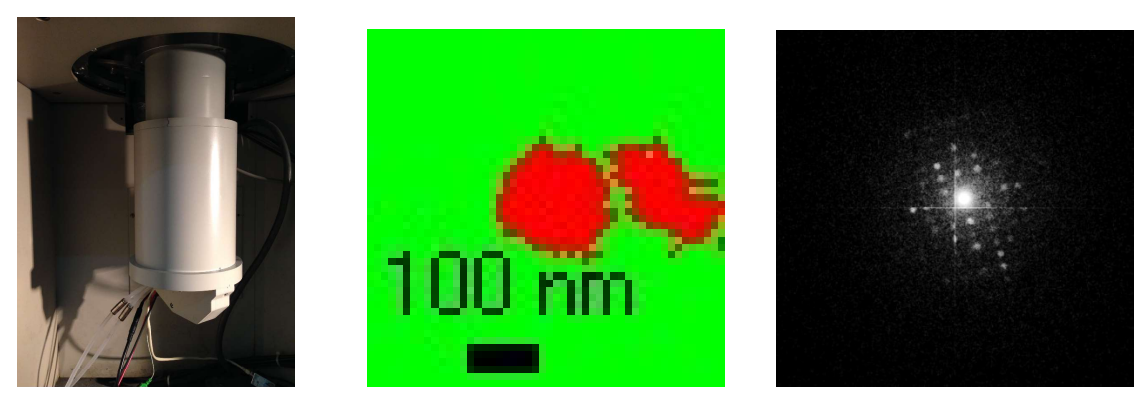

Figure 3. (Left) Timepix detector bottom mounted on a CM30 TEM, (Middle) ASTAR phase mapping where crystalline areas (red) can be readily distinguished from amorphous areas (green) in an ASD mixture, (Right) typical ED pattern from crystalline areas belongs to API. 